\title{
Graphical Site Simulation Using an Object Oriented CAD Model
}

\author{
Toshihiko Aoki, Takahiko Kimura, Kazuhiro Momozaki, Aketo Suzuki \\ Construction Technology Development Department, Taisei Corporation, Sanken Building, \\ 25-1, Hyakunin-cho 3-Chome, Shinjuku-ku, Tokyo 169, Japan
}

\begin{abstract}
Recently, CAD research has concentrated on shifting CAD systems from the traditional line based structure to a more intelligent and object oriented structure. Most work in this area, however, has concentrated on developing applications for design work, leaving construction phase applications largely ignored. The authors of this paper have begun development work on PRISM (Project Representing Intelligent Site Modeler), an intelligent, object oriented CAD tool that will be used during the construction phase as well during design. PRISM will consist of a 3D CAD tool linked to a knowledge base system. The knowledge base will hold various types of site information in the form of object attributes and rules and will be able to reason about the project while CAD will be used to graphically display the results of that reasoning. PRISM will be initially used at the site to represent and simulate retaining wall construction. Readings from inclinometers will be fed directly to PRISM which will graphically display these measurement results. Furthermore, the knowledge base will be able to carry out back analysis and reason about the measurement results, offering suggestions where possible.
\end{abstract}

\section{INTRODUCTION}

Early CAD systems were limited to drawing and drafting by the computer technology which was available at the time. Those CAD systems were essentially electronic drafting tools which were capable of producing only two dimensional drawings. While these graphic presentation techniques revolutionized civil design, they remain largely unused during the actual construction process, the reason being the complexity and vast amounts of information needed and generated at the site. Recent advances in computer technology and CAD technology in particular, however, have given computers the power to represent objects as three dimensional solids and have greatly improved computer response time. Presently, CAD systems are evolving from line based systems to more intelligent object oriented systems that can represent drawings as collections of drawing "objects" capable of holding attributes and behaviors. With advances in computer technology, CAD will expand from design and drafting to the realm of project management and site simulation.

\section{Project Goals}

The authors of this paper have begun development of a new computer tool to be used at the construction site. The tool, PRISM (Project Representing Intelligent Site Modeler) is an object oriented intelligent 3D CAD application composed of a CAD system and a knowledge base system. While object oriented intelligent CAD development has been the topic of research for many years, efforts have concentrated on the design phase usage of such systems. Traditionally, CAD has not been utilized at the site because its only use was as a drawing aid. 
PRISM, however, will be used at the site not to draw, but to display information from the knowledge base. Thus the aim of our research is to develop a comprehensive intelligent CAD system which can be used at the site as well as the design office. Our target is to develop a system that will be able to create a computer model of a project that will follow a project from design to construction. Engineers will add information to the model throughout its existence, making modifications when necessary. The project goals can be categorized into the following four areas:

1) Graphic site representation: The authors would like to present construction data in a graphical, easily understood format. This is especially true for "invisible" information such as rebar strain or soil pressure.

2) Information consolidation: Site information which is presently scattered through various computer applications and log books will be stored in a comprehensive site model.

3) Design and construction integration: Since the site model will be used throughout the life of the project, information from both design and construction will be held by the model. Data, rules, and behaviors pertinent to all construction phases will be recorded in PRISM giving its users a more general concept of the whole project.

4) Store construction knowledge: PRISM will accumulate information in the form of new attributes, rules, or elements as it is used and will act as a storehouse for construction knowledge.

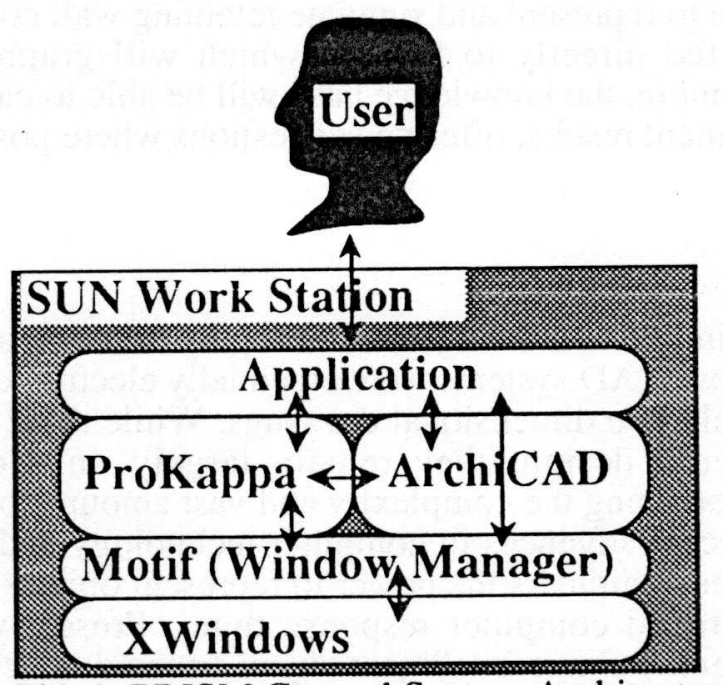

Fig. 1 PRISM General System Architecture

\section{PRISM Architecture}

The CAD system used in PRISM is called ArchiCAD. It is a 3D CAD system developed by Architectural Systems, a Japanese software company. (Please note that this CAD system is not the same ArchiCAD that was developed in the U.S.) The knowledge base system that will hold the project attributes is ProKappa by IntelliCorp from the U.S. The two applications will be connected by a two way dynamic link allowing changes made to the drawing to be registered in ProKappa and allowing reasoning output to be displayed by ArchiCAD. The reason for the two way dynamic link rather than a one way link or an indirect link stems from two 
characteristics of construction work. First, site engineers need immediate feedback to remain up to date on site conditions. This is especially true during emergencies. Second, site conditions are always changing unlike manufacturing where production takes place in a controlled environment.

The overall architecture of PRISM is shown in figure 1. The system runs on a Sun 4 engineering work station using the XWindows graphics platform. The Motif window manager will support ProKappa and ArchiCAD. The user will access the system through an application that provides a graphic user interface.

\section{OBJECT ORIENTED CAD}

Object oriented, when used with reference to CAD, means that lines and shapes are grouped together by function and named to form objects. These objects hold information about the actual object in the form of attributes and rules. The object information in PRISM will be held by the knowledge base. While many CAD packages allow the user to assign attributes to drawings, the attributes are usually limited to text. In object oriented CAD, attributes can be anything from alpha numeric data such as length, type, and material, to rules and behaviors such as the use of the formula $\mathrm{wL}^{2} / 8$ to determine the bending moment at the center of a simply supported beam with a concentrated load. Each object in the drawing is capable of acting independently since each holds its own attributes, rules, and behaviors.

Object oriented CAD offers many advantages over conventional CAD systems. Since drawing objects are capable of holding information, interpretation of the drawing can now be done by computer rather than by humans. Computer will be able to recognize long, "I"-shaped objects in the drawing as I-beams. The implications of this shift from line based CAD to object oriented CAD represents an important advance in CAD technology. CAD diagrams have always contained a wealth of information which was incomprehensible to computer applications without human interpretation. To the computer, a detailed drawing of a construction project would be nothing more that a long list of points and vectors. An engineer at the site would have to look at the drawing, interpret it as a group of objects constituting a construction site, and re-enter the information into various computer tools such as cost management and scheduling software. In the case of an object oriented CAD drawing, computer applications would be able to interpret the drawing and obtain all of the needed information directly. This combination of CAD and knowledge base system results in many benefits which neither application could offer alone.

\section{Modeling Methodology}

The general framework of PRISM is shown in figure 2. Objects are represented in both the knowledge base and CAD. Graphic data about the objects (i.e., shape, size, orientation, and position) are contained in the CAD drawing. Each drawing object also holds an ID number that acts as a pointer to a corresponding instance in the knowledge base. All text data and rules associated with the site objects are held by these instances. By holding non-graphic data in the knowledge base the amount of information carried by the CAD drawing is reduced, making the drawing easier to manipulate.

Element libraries will contain commonly used objects. There will be a number of element libraries, one for each type of construction. Each element in the element library will correspond to a subclass in the knowledge base. For example, if the library consists of anchors, walls, and struts, there will be three corresponding subclasses in the knowledge base called 


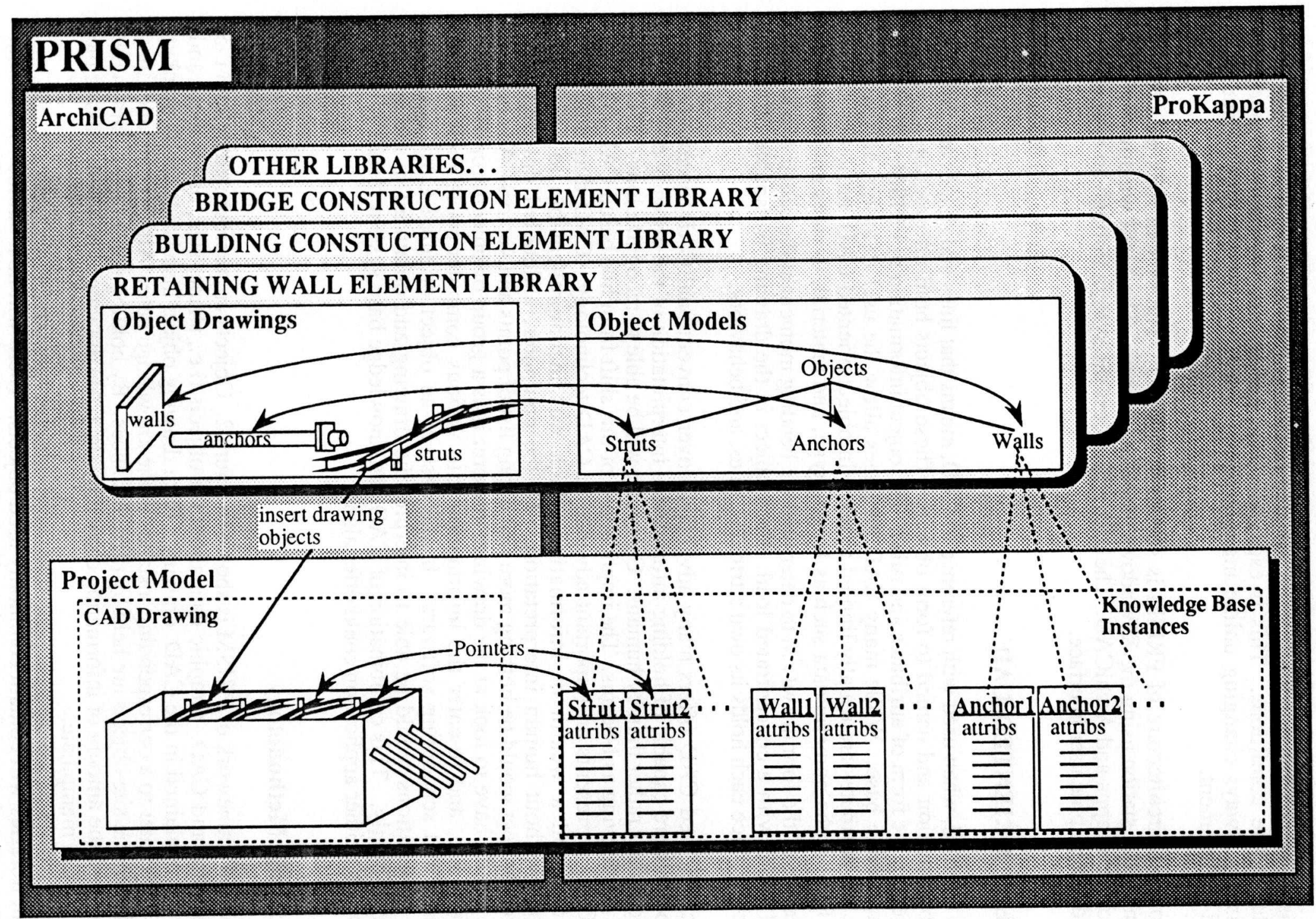

Fig. 2 General Framework of PRISM 
"anchors", "walls", and "struts". During project design, the design engineer will select the desired element library from a library menu and load it into PRISM. To create a site model, a designer simply chooses objects from the menu and inserts them in the drawing. Input is mouse driven wherever possible except for attribute specification which is done by keyboard through dialog boxes. As the objects are placed in the drawing, messages are automatically sent to the knowledge base to create instances under the appropriate sub-classes. Every element inserted into the drawing from the element library will correspond to an instance in the knowledge base.

\section{Retaining Wall Simulation}

While PRISM can be used for a variety of site applications, the authors plan to use it at first to simulate retaining wall construction during excavation work. Usually, when the word simulation is used with reference to construction, it refers to the simulation of a construction process or task for the purpose of feasibility verification or process streamlining. When simulating retaining walls, however, PRISM will be used to simulate ground and wall movements during construction, allowing site engineers to monitor site conditions that cannot be easily visualized.

The typical information flow during retaining wall monitoring is shown in figure 3 . Monitoring can be divided into three main areas: measurement, back analysis, and failure management.

Measurement is relatively simple. Presently, inclinometers and strain gauges at the site feed information to computers which can graphically display the incoming data. The output is usually in the form of two dimensional graphs as shown in figure 4. Although this lets the engineer discern the wall deflections at that point, the graph gives no context. The engineer must use his imagination to determine how the wall deflections relate to the surroundings.

Back analysis is the process of recalculating values assumed during design, such as soil friction angle or cohesiveness, from the actual measured deflections. In retaining wall construction, it is impossible to know all of the pre-existing conditions necessary to analyze wall behavior completely accurately. Assumptions are made concerning soil properties and wall properties during design to predict deflections. During actual construction, deflections are monitored and compared with design values. If excavation is carried out to a certain level and the difference between the design and actual deflection is too great, back analysis is performed. Using values recalculated during back analysis, wall deflections for subsequent excavation work can be predicted at a higher degree of accuracy, thus enhancing site safety. Presently, back analysis systems exist on the computer, although they are stand alone systems.

Failure management is at the core of retaining wall monitoring. In this phase, decisions are made concerning the condition of the site and what actions are required. This task has traditionally been the responsibility of the site engineer because of the reasoning abilities and experience needed.

With both reasoning capabilities provided by ProKappa and presentation capabilities provided by CAD, the authors of this paper hope to integrate these three phases of retaining wall monitoring through the use of PRISM. Measurement data can be collected in the PRISM site model and graphically displayed along with the surrounding area (figure 5). System users will be able to see wall deflections and how those deflections will effect surrounding buildings or even surrounding construction areas. In addition, walls, other supporting members, and 


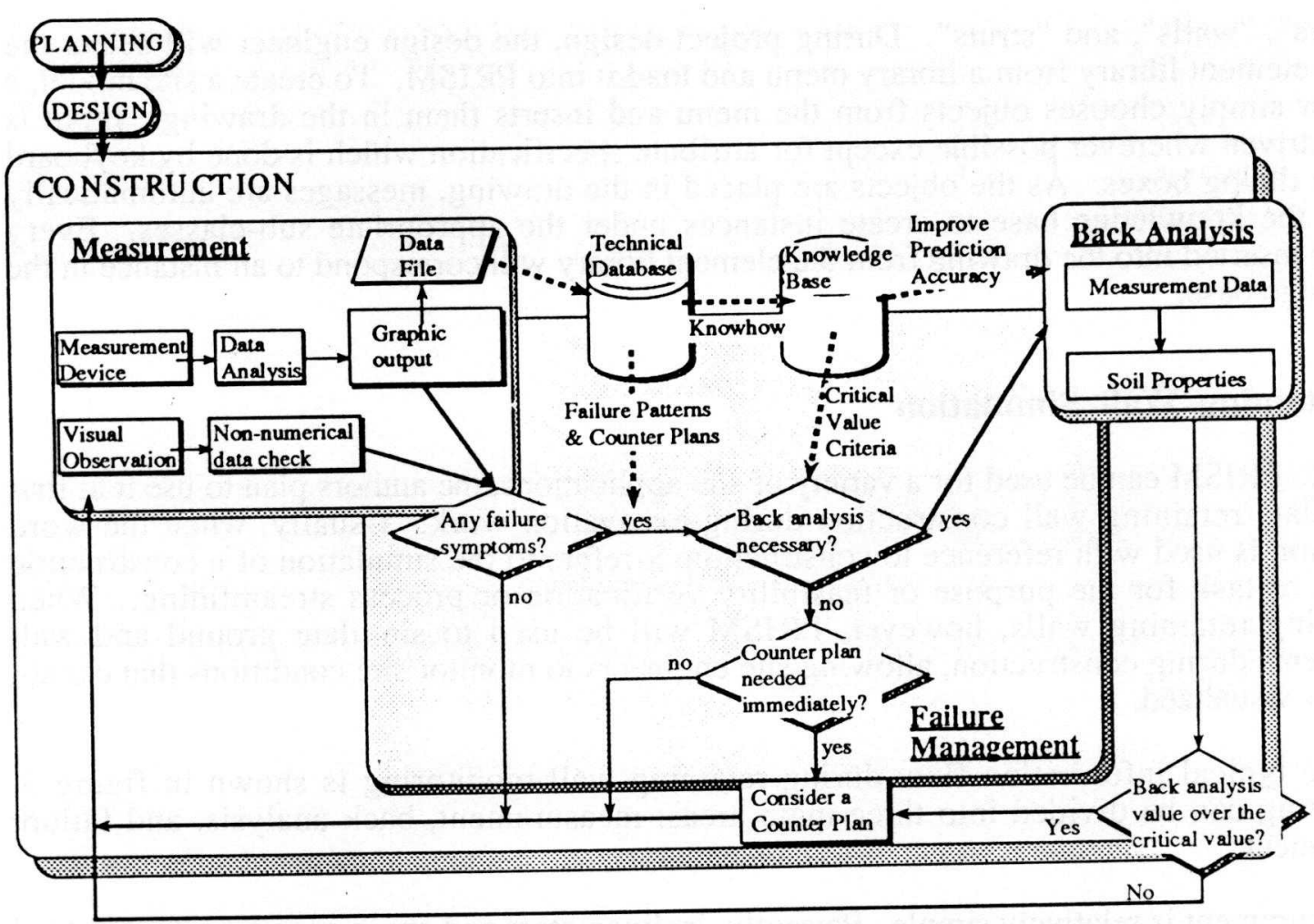

Fig 3 Observational Construction Flow

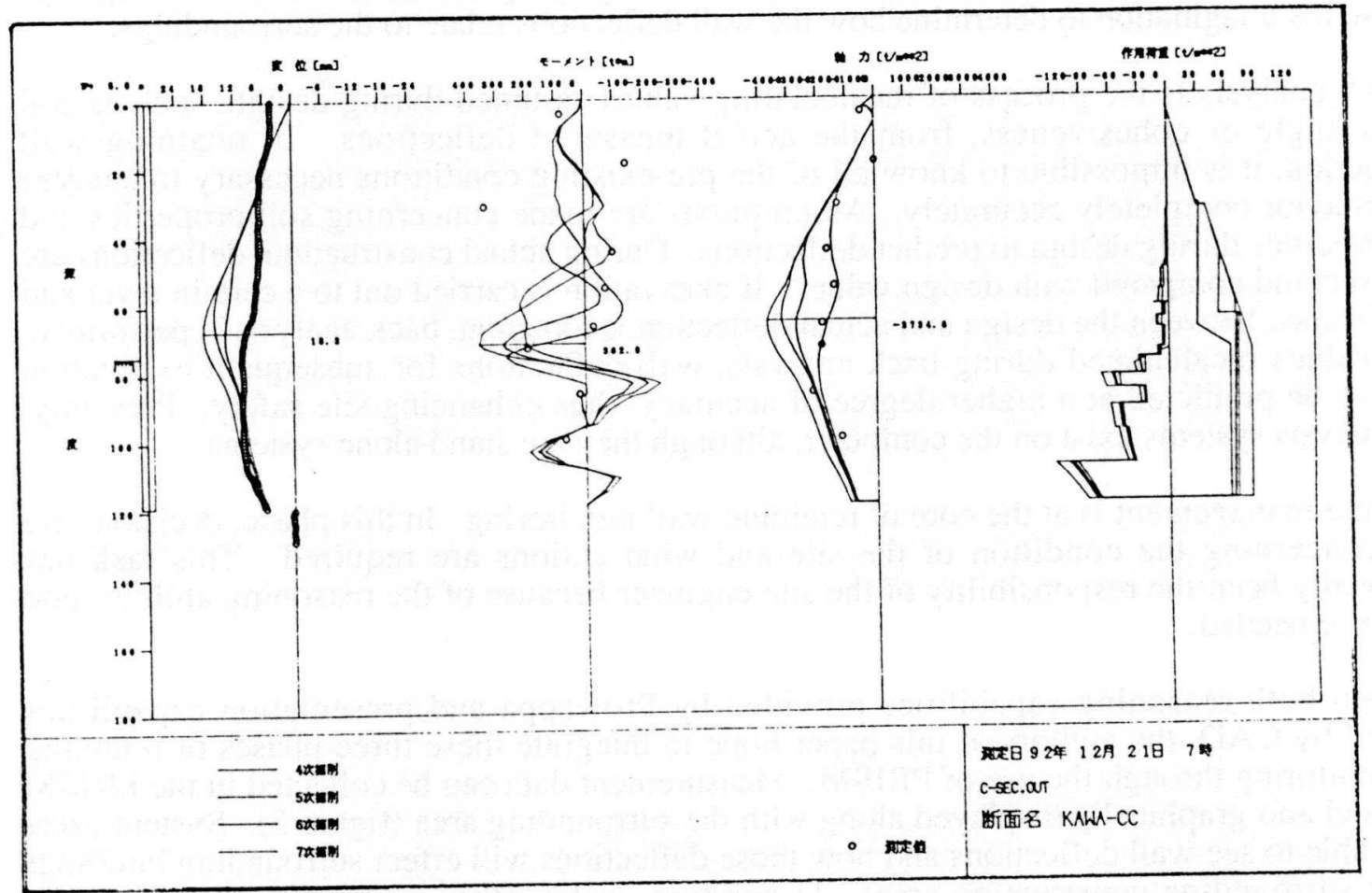

Fig. 4 Two Dimensional Measurement Data Output 


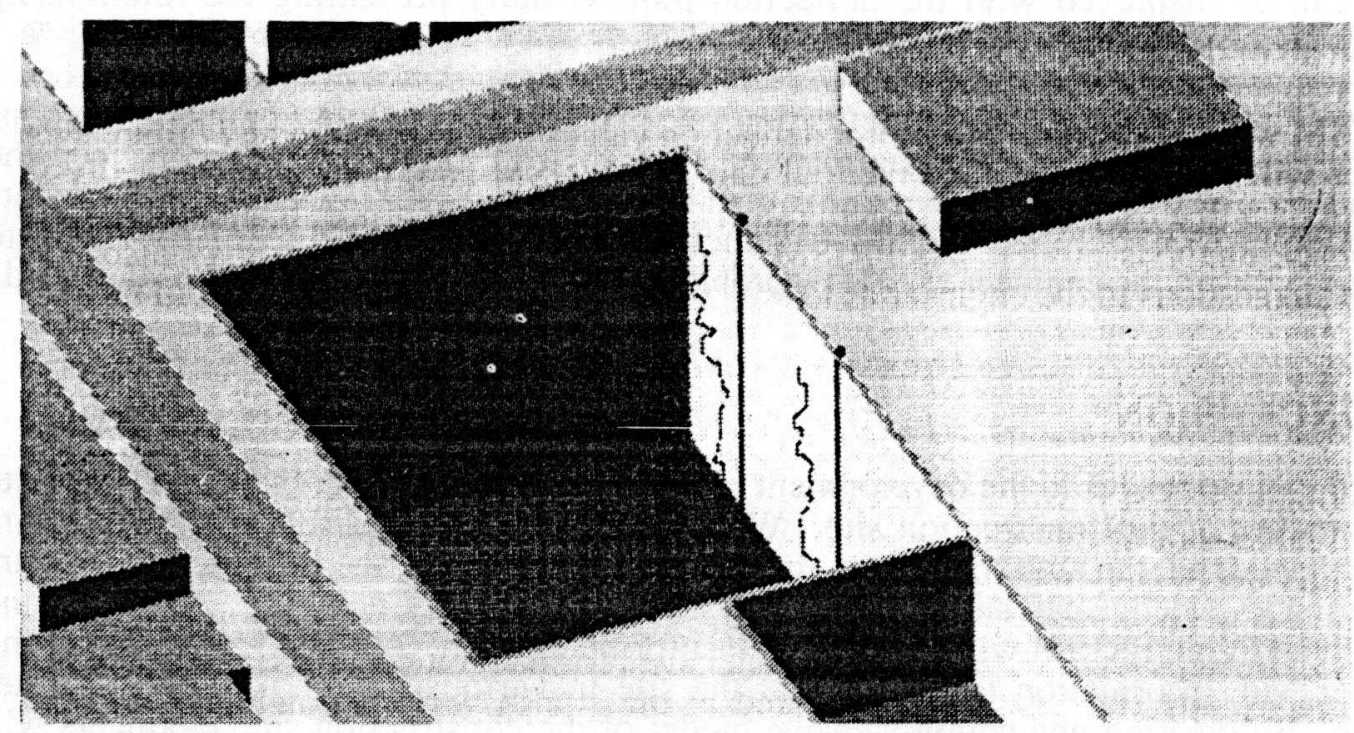

Fig. 5 Three Dimensional Displacement Output

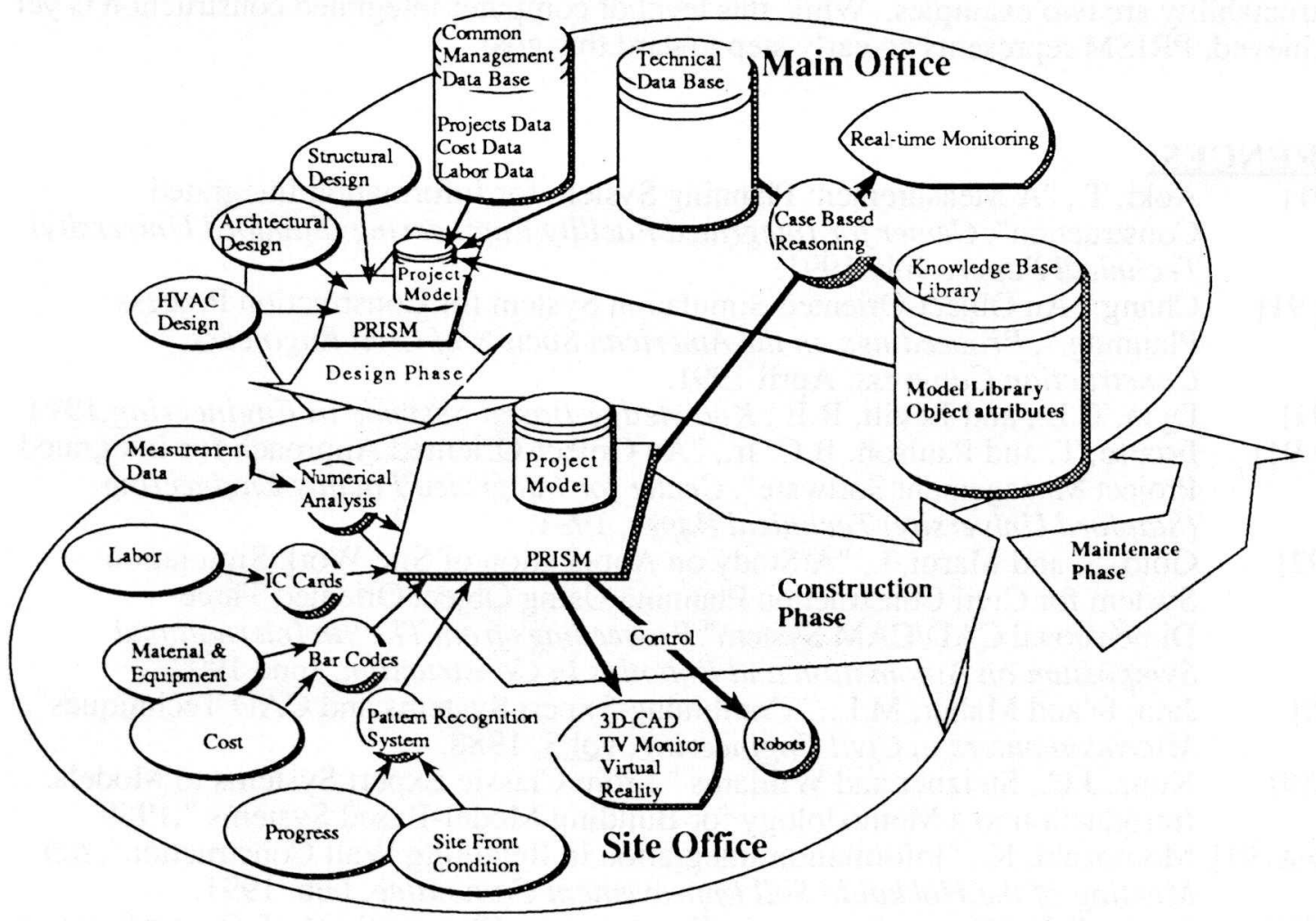

Fig 6 Project Model Life Cycle 
soil layers can be displayed with the deflection plot, visually presenting the relationship between all of these elements.

PRISM will also be able to monitor deflection values and if there are any failure symptoms, the user will be warned of the potential danger. PRISM can, then, automatically feed the pertinent data to a back analysis system rather than having an engineer manually input the data into a back analysis model. As PRISM becomes more developed, it may also be able to provide suggestions to the engineer as to how problems of possible failure could be addressed.

\section{CONCLUSION}

Although PRISM is still in the development phase, the authors believe that it will prove to be a valuable tool at the construction site. When the modeling capabilities of PRISM are sufficiently developed, the authors hope to use it as a storehouse for site information. (Figure 6) Engineering knowledge can be stored in the system in the form of new rules, elements, and attributes. In addition, PRISM will act as a catalyst for integration. As stated at the beginning of this paper, site information is scattered at the site in various notebooks and computer applications. By creating one comprehensive model of the construction site, engineers will have a single information base reducing the need for redundant data. There are two major benefits of integrating separate computer applications. First, information and applications are easier to access when they are located in one area. What is more important, when information from a variety of functions is consolidated in one area, relationships between these functions become more apparent. The influence of structural design on project cost and the requirements of constructability are two examples. While this level of computer integrated construction is yet to be achieved, PRISM represents an early step toward this goal.

\section{REFERENCES:}

[Aoki 91] Aoki, T., "A Measurement Planning System for Information Integrated Construction", Center for Integrated Facility Engineering (Stanford University) Technical Paper, July 1991.

[Chang 91] Chang "An Object-Oriented Simulation System for Construction Process Planning ", Proceedings on the American Society of Civil Engineers Construction Congress, April 1991.

[Dym 91] Dym, C.L., and Levitt, R.E., Knowledge-Based Systems in Engineering, 1991

[Froese 91] Froese, T. and Paulson, B.C. Jr., "An Object-Oriented Approach for Integrated Project Management Software", Center for Integrated Facility Engineering (Stanford University) Technical Paper, 1991.

[Goto 92] Goto, S. and Marui, I., "A Study on Application of Site Work Simulation System for Civil Construction Planning Using Object Oriented ThreeDimensional CAD/CAM System" Proceedings from The 9th International Symposium on Automation and Robotics in Construction, June 1992.

[Jain 88] Jain, D and Maher, M.L., "Combining Expert Systems and CAD Techniques", Microcomputers in Civil Engineering, vol.3, 1988.

[Kunz 89] Kunz, J.C., Stelzner and Williams " From Classic Expert Systems to Models: Introduction to a Methodology for Building Model-Based Systems ",1989

[Momozaki 91] Momozaki, K., "Information Integration in Retaining Wall Construction", 6th Meeting of the Hokkaido Soil Improvement Committee, Feb. 1991.

[Levitt 87] Levitt, R.E.,"Expert Systems in Construction: State of the Art" Expert Systems for Civil Engineers: Technology and application, Chapter 5, 1987.

[Pohl 92] Pohl, J., Myers, L., Cotton J., Chapman, A., Snyder, J., Chauvet, H., Pohl, K.J., and La Porta, J., Design Institute Report CADRU-06-92, 1992. 